Clinical examination is very important in the practice of medicine. In patients presenting with a supraclavicular mass, a number of diseases including cancer should be ruled out. Two patients who presented with a bulky left supraclavicular mass were evaluated. Their medical history revealed complaints attributed to lower urinary tract infection. We performed histopathological examination of the lymph nodes with radiological evaluation of the thorax and abdomen. The final diagnoses were prostate cancer in both patients. It should always be kept in mind that prostate cancer is the most frequent cancer in elderly men, and although very unusual, the presenting finding can be cervical or supraclavicular lymphadenopathy; thus clinicians should be aware of urological examinations in such cases.

Key words: prostate cancer, supraclavicular lymphadenopathy, metastasis.

\section{Metastatic prostate adenocarcinoma presenting as supraclavicular lymphadenopathy: a report of two cases}

\author{
Mürsel Davarci', Ahmet Gokce', E. Oguz Guven', Fatih R. Yalcinkaya', \\ Hasan Esen², Alper Sevinc ${ }^{3}$
}

${ }^{1}$ Mustafa Kemal University Tayfur Ata Sökmen School of Medicine, Department of Urology, Hatay, Turkey

2 Selçuk University School of Medicine, Department of Pathology, Konya, Turkey ${ }^{3}$ Gaziantep University, Gaziantep Oncology Hospital, Department of Medical Oncology, Gaziantep, Turkey

\section{Introduction}

Prostate cancer is the sixth most frequent cancer in the world (in the number of new cases), the third most common cancer in men, and the most frequent cancer in men in Europe, North America, and some parts of Africa [1]. The most general sites of prostate cancer distant metastasis are bones, regional lymph nodes, lung, liver, brain, and the epidural space [2]. However, metastasis from prostate cancer is an extremely unusual situation with a reported rate of $0.4 \%$ to $1 \%$ of cases [3]. We report two prostate cancer cases that presented with a supraclavicular bulky mass as the initial and the most bothersome symptom.

\section{Case 1}

A 78-year-old male who attended the emergency department with a bulky left supraclavicular mass was consulted because of lower urinary tract symptoms. Physical examination revealed a solid, multilobulated mass in the left supraclavicular region, measuring $9 \mathrm{~cm}$ at its longest diameter. He reported weight loss of $15 \mathrm{~kg}$ over the previous four months and symptoms related to lower urinary tract obstruction for five months.

Laboratory investigations in terms of whole blood count, blood chemistry and urinalysis were all in the normal ranges. A thorough genitourinary examination revealed no obvious pathology. Digital rectal examination (DRE) revealed a hard, irregular prostate with a volume of $58 \mathrm{cc}$ in transrectal ultrasonography (TRU). Serum prostate-specific antigen (PSA) level was $171.0 \mathrm{ng} / \mathrm{ml}$. Bone scintigraphic examination revealed a metastatic lesion at the fifth lumbar vertebra.

Fine needle biopsy of the left-sided supraclavicular lymph node was performed. Histological examination revealed a lymph node widely replaced by metastatic adenocarcinoma staining positive for PSA and prostate-specific acid phosphatase consistent with metastatic adenocarcinoma of the prostate (Fig. 1). Therefore, twelve quadrant fine needle biopsies were performed under TRU guidance which revealed a prostate cancer with a Gleason score of 8/10.

He was started on cyproterone acetate, $100 \mathrm{mg}$, three times a day and leuprorelin acetate injections, $3.75 \mathrm{mg}$, monthly. Ten months later, the patient had significant symptomatic relief with a marked reduction in supraclavicular lymphadenopathy and a decreased prostate-specific antigen level of $12.1 \mathrm{ng} / \mathrm{ml}$. 


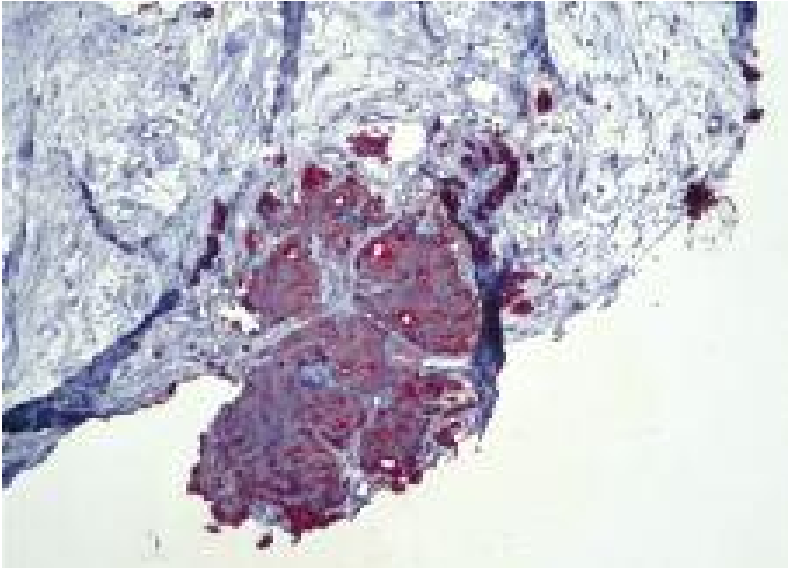

Fig. 1.

\section{Case 2}

A 65-year-old male patient was admitted to the oncology outpatient clinic with complaints of left supraclavicular lymphadenopathy. Physical examination revealed a solid, $5 \mathrm{~cm}$ mass in the left supraclavicular region.

Laboratory investigations in terms of whole blood count, blood chemistry and urinalysis were all in the normal ranges. A thorough genitourinary examination revealed no obvious pathology. Digital rectal examination (DRE) revealed a hard, irregular prostate with a volume of $64 \mathrm{cc}$ in transrectal ultrasonography (TRU). Serum prostate-specific antigen (PSA) level was $>1500 \mathrm{ng} / \mathrm{ml}$. Serum free PSA level was $>25 \mathrm{ng} / \mathrm{ml}$. Bone scintigraphic examination revealed multiple vertebral metastatic lesions.

Trucut biopsy of the left-sided supraclavicular lymph node was performed. Histological examination revealed a metastatic adenocarcinoma staining positive for PSA and prostatespecific acid phosphatase consistent with metastatic adenocarcinoma of the prostate. He was started on bicalutamide 50 mg once a day.

\section{Discussion}

Prostate cancer is the most frequent cancer and the second leading cause of cancer death in men [4]. Dissemination of prostate cancer into the adjacent organs (urethra, bladder neck and seminal vesicles) is rare [5]. Prostate carcinoma is known to invade by way of three mechanisms: local, haematogenous spreading, and lymphatic metastasis [6]. Bone represents the principal location of distant metastasis in prostate cancer [7]. Lymphatic spreading occurs most often in regional lymph nodes such as the obturator, internal and external iliac lymph nodes, presacral and para-aortic lymph nodes. Distant superficial lymphadenopathy is a rare symptom on initial presentation in prostate cancer [8].

In terms of a supraclavicular mass discovered during physical examination, most frequently nasopharynx, oral cavity and upper gastrointestinal tract cancer metastases to cervical and supraclavicular lymph nodes are observed [9-11].

Saeter et al. [12] noted that, in 35 patients with non-regional lymphatic invasion from prostate carcinoma, the left supraclavicular fossa was the most common location of metastasis in $69 \%$ of cases. Butler et al. published 19 patients with prostate cancer presenting at first with supraclavicular lymphadenopathy, in which the diagnosis was verified by prostate biopsy in 14 patients. They reported that only $42 \%$ of all patients had an abnormal DRE [13]. Case 1 also had an abnormal DRE. Woo et al. described a 76-year-old patient presenting with supraclavicular lymphadenopathy. They noted a normal prostate on DRE. A PSA performed a few days after admission was $326 \mathrm{ng} / \mathrm{ml}$ and a fine needle biopsy of the lymphadenopathy confirmed a prostate cancer metastasis [14]. Cho et al. reported 26 cases of metastatic prostate cancer in supradiaphragmatic lymph nodes, in which only 7 cases had a history of prostate cancer; they noted that $58 \%$ had an abnormal rectal examination [15].

Although prostate cancer is widespread, the prostate is often overlooked as the first site for men presenting with supraclavicular lymph node metastases [5]. Fine needle aspiration biopsy of supraclavicular lymph nodes may be useful for pathological diagnosis of prostate cancer metastasis. In our patients, serum PSA level and immunohistochemical staining for PSA in biopsy material were used in detection of the primary location of the cancer. Two patients had highly elevated serum PSA levels, and bone scintigraphic examination revealed metastatic lesions.

It should always be kept in mind that prostate cancer is the most frequent cancer in elderly men, and although very unusual, the presenting finding can be a cervical or supraclavicular lymphadenopathy; thus clinicians should be aware of urological examinations of such cases.

\section{References}

1. Gronberg H. Prostate cancer epidemiology. Lancet 2003; 361: 859-64.

2. Oesterling J, Fuks Z, Lee C, Scher HI. Cancer of the prostate. In: Cancer: Principles and Practise of Oncology. Devita V, Hellman S, Rosenberg S (eds.). Edition 5th. Lippincott-Raven, Philadelphia $1997 ; 1322-86$

3. Jones H, Anthony PP. Metastatic prostatic carcinoma presenting as left-sided cervical lymphadenopathy: a series of 11 cases. Histopathology 1992; 21: 149-54.

4. Coffey D. Prostate cancer: an overview of an increasing dilemma. Cancer 1993; 71: 880-6.

5. Dick VS. Carcinoma of the prostate gland with metastases. Surg Clin North Am 1962; A2: 771-7.

6. Venable DD, Hastings D, Misra RP. Unusual metastatic patterns of prostate adenocarcinoma. J Urol 1983; 130: 980-5.

7. Corriere JN, Cornog JL, Murphy JJ. Prognosis in patients with carcinoma of the prostate. Cancer 1970; 25: 911-8.

8. Catalona WJ, Scott WW. Carcinoma of the prostate. In: Campell's Urology. Harrison JH, Gittes RF, Perlmutter AD (eds.). WB Saunders, Philedelphia 1979; 1085.

9. Basso L, Izzo L, Calisi E, et al. Cervical node metastasis as the first sign of cancer of the caecum. Anticancer Res 2007; 27: 3589-92.

10. Chitale SV, Harry L, Gaches CG, Ball RY. Presentation of prostatic adenocarcinoma with cervical lymphadenopathy: two case reports and review of the literature. Otolaryngol Head Neck Surg 2001; 125: 431-2.

11. Clark S, Sanderson RJ, McLaren KM. Metastatic prostatic carcinoma presenting as cervical lymphadenopathy. J Oral Maxillofac Surg 2001; 59: 571-3.

12. Saeter G, Fosså SD, Ous S, Blom GP, Kaalhus O. Carcinoma of the prostate with soft tissue or non-regional lymphatic metastases at the time of diagnosis: a review of 47 cases. Br J Urol 1984; 56: 385-90.

13. Butler JJ, Howe C, Johnson D. Enlargement of supraclavicular lymph nodes as the initial sign of prostate carcinoma. Cancer 1971; 27: 1055-63. 
14. Woo K, Wieczorek R, Torre P, Lepor H. Prostate adenocarcinoma presenting as a large supraclavicular mass. Rev Urol 2001; 3: 102-5.

15. Cho KR, Epstein Jl. Metastatic prostatic carcinoma to supradiaphragmatic lymph nodes. A clinicopathologic and immunohistochemical study. Am J Surg Pathol 1987; 11: 457-63.

\section{Address for correspondence}

Mürsel Davarci MD, Assist Prof.

Department of Urology, Faculty of Medicine,

Mustafa Kemal University

31100 Hatay, Turkey

tel. +90-326 2291000 (Ext. 2917)

fax: +90-326 2455305

e-mail: mdavarci1970@hotmail.com

Submitted: $\quad$ 7.04.2011

Accepted: $\quad$ 18.01.2012 